\title{
Pediatric Case Report
}

\section{Ureteral Quintuplication With Renal Atrophy in an Infant After the 1986 Chernobyl Nuclear Disaster}

\section{Beata Jurkiewicz, Tomasz Ząbkowski, and Dmitrij Shevchuk}

Ureteral duplication is a comparatively frequent urinary tract anomaly. Ureteral triplication is rare, but quadruplication is extremely rare. In this study, we describe a case of ureteral quintuplication, the first such report in the Englishlanguage literature. A newborn female baby was diagnosed with left ureteral quintuplication. The left ureter was divided into 5 ureters with 5 renal pelvises within approximately $3 \mathrm{~cm}$ of the urinary bladder, and trace parenchyma of the kidney was noted. The patient was born within $60 \mathrm{~km}$ of the epicenter of the 1986 Chernobyl disaster, 24 years after the catastrophic nuclear accident, and is currently aged 3 years. UROLOGY 83: 211-213, 2014. (c) 2014 Elsevier Inc.

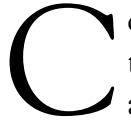
ongenital anomalies of the kidney and urinary tract constitute approximately $20 \%-30 \%$ of all anomalies identified in the prenatal period. ${ }^{1}$ Defects can be bilateral or unilateral, and different defects often coexist in a single child.

Congenital anomalies of the kidney and urinary tract occur at a frequency of 1 in 500 live births and are a common cause of renal insufficiency in childhood. They encompass a wide spectrum of malformations, including anomalies of the kidney, collecting system, bladder, and urethra. ${ }^{2}$ Worldwide statistics indicate that they are a cause of end-stage renal failure in children in 30\%-43\% of cases, ${ }^{3}$ and the rate increases to $60 \%$ when the earlier stages of chronic kidney disease are considered. ${ }^{4}$ Although ureteral duplication is a comparatively frequent urinary tract anomaly, identified in $0.32 \%-0.8 \%$ of the population, triplication is rare, and quadruplication is extremely rare. Until 2001, only 5 cases were described in the English-language literature, most of which involved incomplete multiplication and associated additional urinary tract malformations. In this study, we report a case of ureteral quintuplication and present postoperative photos. This is the first such report in the Englishlanguage literature.

\section{CASE REPORT}

The patient is a 3-year-old girl who was born within $60 \mathrm{~km}$ of the epicenter of the 1986 Chernobyl disaster (Ukraine), 24 years after the catastrophic nuclear accident. The mother lived in Owrucz, within $60 \mathrm{~km}$ of

Financial Disclosure: The authors declare that they have no relevant financial interests. From the Department of Pediatric Surgery, Children's Hospital in Dziekanow Lesny, Warsaw, Poland; the Department of Urology, Military Institute of Medicine, Warsaw, Poland; and the Department of Pediatric Surgery, Zhytomyr Regional Child's Hospital, Ukraine

Reprint requests: Tomasz Zabkowski, M.D., Department of Urology, Military Institute of Medicine, Szaserow 128, 00-909 Warsaw, Poland. E-mail: urodent@wp.pl

Submitted: May 28, 2013, accepted (with revisions): July 22, 2013
Chernobyl, during her pregnancy and was in the area of the epicenter when the urinary tract system of the infant would have developed.

Prenatal ultrasound scans at 15-16 HBD (ie, week of pregnancy; from the Latin, hebdomen) showed urine retention in the right kidney of the fetus. Repeat ultrasound at $34 \mathrm{HBD}$ showed malformation syndrome consistent with polycystic disease of the right kidney and digestive tract pathology of unknown etiology. Caesarean section was performed at 36 weeks' gestational age because of intrauterine infection and fetal asphyxia. The infant's birth weight was $2380 \mathrm{~g}$, and the Apgar score was 1. After birth, the baby was admitted to the intensive care unit, where antibiotics were administered and diagnosis of malformations was initiated. Postnatal diagnoses included oesophageal atresia with tracheo-oesophageal fistula, biliary atresia, hydronephrosis of the right kidney and right megaloureter, persistent foramen ovale in interatrial septum, and anemia. Genetic studies were not performed.

Surgery was performed on day 2, consisting of thoracotomy with end-to-end anastomosis of the esophagus and closure of the tracheo-oesophageal fistula (medical history number - 1482/2010). The patient received antibiotics, including cephalosporins, amikacin, vancomycin, and fluconazole. Postnatal ultrasound showed an enlarged right kidney measuring $55 \times 26 \mathrm{~mm}$, calyces of $2 \mathrm{~mm}$, parenchyma of $13 \mathrm{~mm}$, renal pelvis measuring $20 \times 9 \mathrm{~mm}$, the right ureter in perivesical space with a width of $8 \mathrm{~mm}$, left kidney measuring $30 \times 14 \mathrm{~mm}$. The renal pelvis and ureter were undetectable.

The patient was diagnosed with acute pyelonephritis at 5 months. Urine cultures showed Pseudomonas aeruginosa infection, and the patient was treated with Fortum, Meronem, Flonazol, and Furagin. She was hospitalized many times during the first year of life because of urinary tract infections. Micturating cystourethrogram revealed grade $\mathrm{V}$ right vesicoureteral reflux and grade II left vesicoureteral reflux. The patient was not on antibiotic 


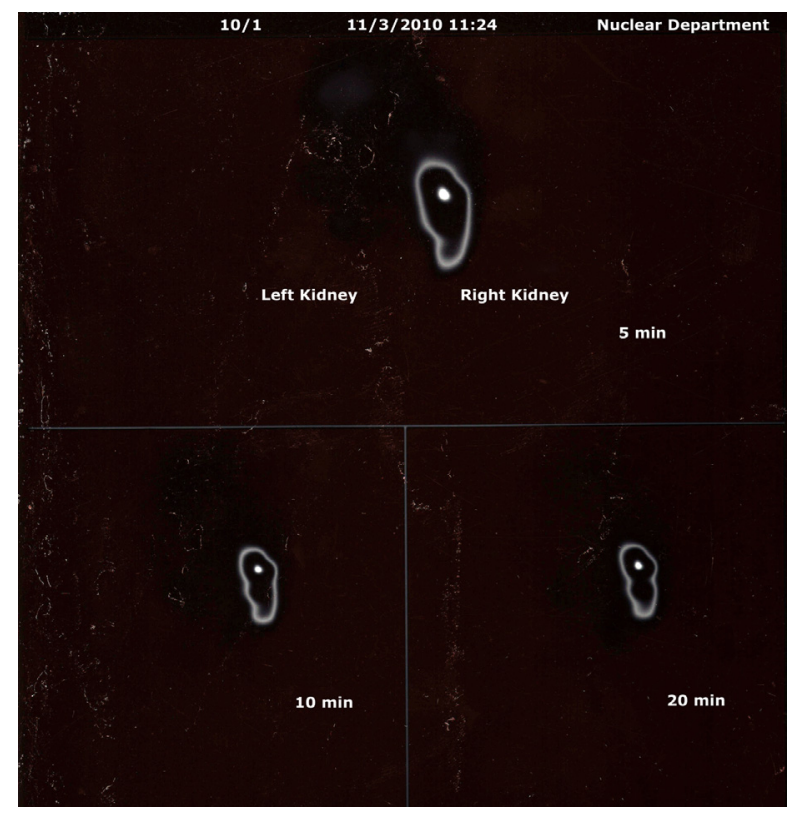

Figure 1. Renal scintigraphy - nonexcretion in the left kidney. (Color version available online.)

prophylaxis after vesicoureteral reflux was diagnosed because there are not these recommendations in Ukraine. Isotope study showed trace renal excretion and impaired emptying of the right kidney. Because of urine retention in the right upper urinary tract and nonexcretion in the left kidney, ureterocutaneostomy was performed on the right side to improve drainage of urine. In 2010, the patient underwent operation by William's technique (medical history number - 13620/2010). During this operation, ureter fissus was diagnosed. The ureterocutaneostomy was installed below the ureteral bifurcation receiving good vesicoureteral reflux of the right kidney and nonretention on this side. The patient was on antibiotic prophylaxis with Nitrofurantoin in dose of $2 \mathrm{mg} / \mathrm{kg} /$ day. However, the urinary tract infections persisted, and the patient was hospitalized many times. Urine cultures indicated infection with Escherichia coli, Enterococcus faecalis, Streptococcus pyogenes, and $P$ aeruginosa. Repeat isotope study of the kidney confirmed nonexcretion in the left kidney (Fig. 1). Left nephrectomy was elected (medical history number - 14421/2011) and performed without complications. The complex renal anomaly of the left kidney was diagnosed during the operation (Fig. 2). Quintuplication of the left ureter was identified, specifically division into 5 ureters with 5 renal pelvises within approximately $3 \mathrm{~cm}$ of the urinary bladder (Fig. 3). Trace parenchyma of the kidney was also noted. Everything was ablated without complications. Cephalosporin was administered after the surgery. Currently, no urinary tract infections were diagnosed in the baby after left nephrectomy with well-functioning ureterocutaneostomy on the right side. Creatinine and urea levels were normal. Control isotope study of the kidney was performed in December 2012 and showed progression in secretory and excretory function.

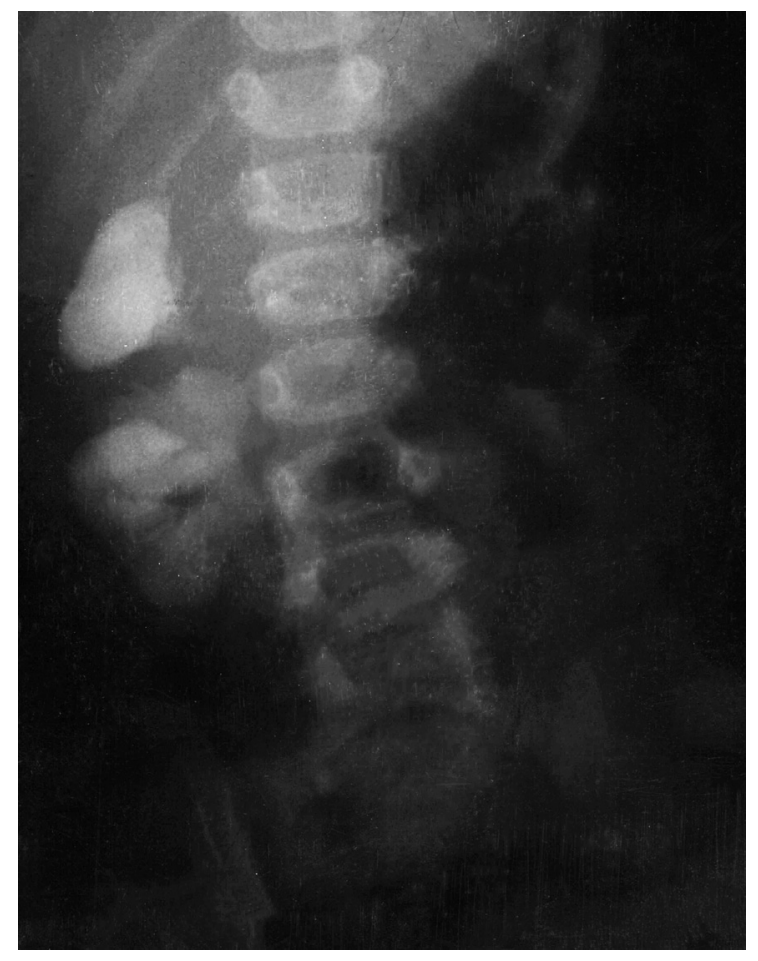

Figure 2. Urography - hydronephrosis of duplex right kidney. Nonexretion of the left kidney.

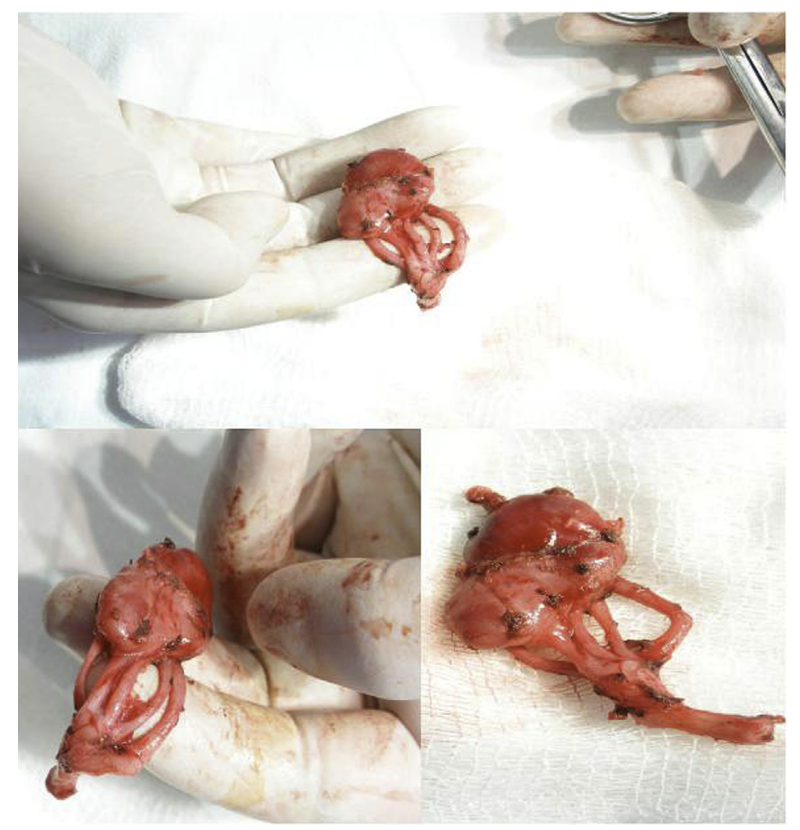

Figure 3. The surgical specimen - the left kidney with 5 ureters and 5 renal pelvises. (Color version available online.)

\section{COMMENT}

Congenital malformations of the kidney and urinary tract account for $50 \%$ of all congenital abnormalities, and approximately $85 \%$ of these involve the urinary tract. ${ }^{5}$ The ureters develop at the beginning of the 5 th week 
of gestation as ureteral buds (UBs) originating from the mesonephric (Wolffian) duct. A series of inductive interactions occur in the intermediate mesoderm, between the metanephric mesenchyme and the epithelial derivatives of UB at the caudal end of the nephrogenic cord. The UBs grow rapidly to penetrate the metanephric mesenchyme and give rise to the renal collecting system, that is, the ureters, renal pelvises, calyces, papillary ducts, and collecting ducts. ${ }^{6}$ Embryologyically, supernumerary ureters can arise because of duplication of the ureteric bud, ${ }^{6}$ a process, which can be partial or complete. The metanephric tissue may then divide into multiple parts, each with its own pelvis and ureter. ${ }^{7}$ Ureteral duplication as a common urinary tract malformation has been precisely described. Ureteral triplication is a rare malformation, and only approximately 100 triplication cases have been reported. ${ }^{8}$ We searched the English-language literature and found only 5 cases of ureteral quadruplication and 1 case of 6 functioning kidneys on the left side. ${ }^{9-13}$ Our case is the first of ureteral quintuplication with associated renal atrophy. In our patient, the left ureter divided into 5 ureters with 5 renal pelvises within approximately $3 \mathrm{~cm}$ of the urinary bladder and trace renal parenchyma was noted. The patient was born within $60 \mathrm{~km}$ of the 1986 Chernobyl disaster epicenter, 24 years after the catastrophic nuclear accident. The Chernobyl disaster is widely considered to have been the worst nuclear power plant accident in history and is one of only 2 classified as a level 7 event on the International Nuclear Event Scale, the other being the Fukushima Daiichi nuclear disaster in 2011. ${ }^{14}$ Environmental factors such as viral and bacterial infections, $\mathrm{x}$-ray radiation exposure, drugs, and smoking are significant etiologic factors in congenital anomalies of the kidney and urinary tract. Environmental factors such as viral and bacterial infections, X-ray radiation exposure, drugs, smoking, alcohol abuse, food shortage, and pharmaceuticals are significant aetiologic factors in congenital anomalies of the kidney and urinary tract. ${ }^{2}$

We provide charitable surgical services to patients at Zhytomyr Regional Children's Hospital. This hospital has 430 beds and a single $\mathrm{x}$-ray unit dating from 1957. Under such difficult diagnostic conditions, the quality of the photographs is less than optimal.

\section{References}

1. Queisser-Luft A, Stolz G, Wiesel A, et al. Malformations in newborn: results based on 30,940 infants and fetuses from the Mainz congenital birth defect monitoring system (1990-1998). Arch Gynecol Obstet. 2002;266:163-167.

2. Zwolińska D, Polak-Jonkisz D, Makulska I. Genetics of congenital anomalies of the kidney and urinary tract. Postepy Hig Med Dosw. 2011;65:829-837.

3. Song R, Yosipiv IV. Genetics of congenital anomalies of the kidney and urinary tract. Pediatr Nephrol. 2011;26:353-364.

4. Harambat J, van Stralen KJ, Kim JJ, Tizard EJ. Epidemiology of chronic kidney disease in children. Pediatr Nephrol. 2012;27:363-373.

5. Sadler TW. Langman's Medical Embryology. Williams and Wilkins; Baltimore: 1995;272-311.

6. Baker LA, Gomez RA. Embryonic development of the ureter. Semin Nephrol. 1998;18:569-584.

7. Quaggin SE, Kreidberg J. Embryology of the kidney. In: Brenner and Rector's The Kidney. Saunders Elsevier; 2008:6-14.

8. Kudela G, Koszutski T, Mikosinski M, et al. Ureteral triplication report of four cases. Eur J Pediatr Surg. 2006;16:279-281.

9. Klinge L, Kubiak R, Slongo T. Quadriplication of the ureter in combination with a large ureteral cyst. Pediatr Surg Int. 2001;17: 566-568.

10. Lopes RI, Lopes RN, Filho CMB. Ureteral quadriplication with contralateral triplicate ureter. J Urol. 2001;166:979-980.

11. Bolkier M, Moskovitz B, Ginesin Y, et al. Incomplete quadriplication of urinary tract with contralateral agenesis of the kidney. Eur Urol. 1991;19:267-268.

12. Begg RC. Sextuplicitas renum: a case of six functioning kidneys and ureters in an adult female. J Urol. 1953;70:686-693.

13. Huang CJ. Congenital giant megaureter. J Pediatr Surg. 1987;22: 235-239.

14. Black R, (12 April 2011), "Fukushima: As Bad as Chernobyl?", BBC, Retrieved 20 August 2011 